\title{
SOME SANDWICH-TYPE RESULTS FOR $\phi$-LIKE FUNCTIONS
}

\author{
P. Kaur, S. Singh Billing
}

ABStract. Using the technique of differential subordination, we here obtain certain results for $\phi$-like, starlike and close-to-convex functions.

2010 Mathematics Subject Classification: 30C45.

Keywords: analytic function, $\phi$-like functions, starlike function, differential subordination, differential superordination.

\section{INTRODUCTION}

Let $\mathcal{H}$ be the class of functions analytic in $\mathbb{E}=\{z:|z|<1\}$ and $\mathcal{H}[a, n]$ be the subclass of $\mathcal{H}$ consisting functions of the form

$$
f(z)=a+a_{n} z^{n}+a_{n+1} z^{n+1}+\cdots .
$$

Let $\mathcal{A}$ be the subclass of $\mathcal{H}$ consisting of functions $f$, analytic in the open unit disk $\mathbb{E}=\{z:|z|<1\}$ and normalized by the conditions $f(0)=f^{\prime}(0)-1=0$. A function $f \in \mathcal{A}$ is said to be starlike of order $\beta, 0 \leq \beta<1$, if and only if

$$
\Re\left(\frac{z f^{\prime}(z)}{f(z)}\right)>\beta, z \in \mathbb{E} .
$$

The class of such functions is denoted by $\mathcal{S}^{*}(\beta)$. Note that $\mathcal{S}^{*}(0)=\mathcal{S}^{*}$, the class of univalent starlike functions.

A function $f \in \mathcal{A}$ is said to be close-to-convex in $\mathbb{E}$ if it satisfies the condition

$$
\Re\left(\frac{z f^{\prime}(z)}{g(z)}\right)>0, z \in \mathbb{E}, \text { for } g \in \mathcal{S}^{*} .
$$

The class of close-to-convex functions is denoted by $\mathcal{C}$. Noshiro [2] and Warchawski [6] independently proved in 1934-35 that $f$ is close-to-convex if

$$
\Re\left(f^{\prime}(z)\right)>0 .
$$


Let $\Phi: \mathbb{C}^{2} \times \mathbb{E} \rightarrow \mathbb{C}$ be an analytic function, $p$ be an analytic function in $\mathbb{E}$ with $\left(p(z), z p^{\prime}(z) ; z\right) \in \mathbb{C}^{2} \times \mathbb{E}$ for all $z \in \mathbb{E}$ and $h$ be univalent in $\mathbb{E}$. Then the function $p$ is said to satisfy first order differential subordination if

$$
\Phi\left(p(z), z p^{\prime}(z) ; z\right) \prec h(z), \quad \Phi(p(0), 0 ; 0)=h(0) .
$$

A univalent function $q$ is called a dominant of the differential subordination (1) if $p(0)=q(0)$ and $p \prec q$ for all $p$ satisfying (1). A dominant $\tilde{q}$ that satisfies $\tilde{q} \prec q$ for all dominants $q$ of (1) is said to be the best dominant of (1).

Let $\Psi: \mathbb{C}^{2} \times \mathbb{E} \rightarrow \mathbb{C}$ be analytic and univalent in domain $\mathbb{C}^{2} \times \mathbb{E}, h$ be analytic in $\mathbb{E}, p$ be analytic and univalent in $\mathbb{E}$, with $\left(p(z), z p^{\prime}(z) ; z\right) \in \mathbb{C}^{2} \times \mathbb{E}$ for all $z \in \mathbb{E}$. Then $p$ is called a solution of the first order differential superordination if

$$
h(z) \prec \Psi\left(p(z), z p^{\prime}(z) ; z\right), h(0)=\Psi(p(0), 0 ; 0) .
$$

An analytic function $q$ is called a subordinant of the differential superordination (2), if $q \prec p$ for all $p$ satisfying (2). A univalent subordinant $\tilde{q}$ that satisfies $q \prec \tilde{q}$ for all subordinants $q$ of (2) is said to be the best subordinant of (2).

The function $f \in \mathcal{A}$ is called $\phi$-like in the open unit disk $\mathbb{E}$, if

$$
\Re\left(\frac{z f^{\prime}(z)}{\phi(f(z))}\right)>0, z \in \mathbb{E},
$$

where $\phi$ is analytic in a domain containing $f(\mathbb{E}), \phi(0)=0=\phi^{\prime}(0)-1$ and $\phi(w) \neq 0$ for $w \in f(\mathbb{E}) \backslash\{0\}$. This concept was first introduced by Brickman [1] and he established that a function $f \in \mathcal{A}$ is univalent if and only if $f$ is $\phi$-like for some $\phi$.

Using the concept of differential subordination Ruscheweyh [9] introduced and studied the following more general class of $\phi$-like functions:

Let $\phi$ be analytic function in the domain containing $f(\mathbb{E}), \phi(0)=0=\phi^{\prime}(0)-1$ and $\phi(w) \neq 0$ for $w \in f(\mathbb{E}) \backslash\{0\}$. Then $f \in \mathbb{A}$ is called $\phi$-like w.r.t. a univalent function $q(z)$ if $\frac{z f^{\prime}(z)}{\phi(f(z))} \prec q(z), z \in \mathbb{E}$.

In 2005, Ravichandran et al.[10] proved the following result for $\phi$-like functions:

Let $\alpha \neq 0$ be a complex number and $q(z)$ be a convex univalent function in $\mathbb{E}$. Suppose $h(z)=\alpha q^{2}(z)+(1-\alpha) q(z)+\alpha z q^{\prime}(z)$ and

$$
\Re\left\{\frac{1-\alpha}{\alpha}+2 q(z)+\left(1+\frac{z q^{\prime \prime}(z)}{q^{\prime}(z)}\right)\right\}>0, z \in \mathbb{E} .
$$

If $f \in \mathcal{A}$ satisfies

$$
\frac{z f^{\prime}(z)}{\phi(f(z)}\left(1+\frac{\alpha z f^{\prime \prime}(z)}{f^{\prime}(z)}+\frac{\alpha\left(f^{\prime}(z)-(\phi(f(z)))^{\prime}\right.}{\phi(f(z))}\right) \prec h(z)
$$


then

$$
\frac{z f^{\prime}(z)}{\phi(f(z))} \prec q(z), z \in \mathbb{E}
$$

and $q(z)$ is best dominant.

Recently, Shanmugam et al. [5] and Ibrahim [3] also obtained the results for $\phi$-like functions parallel to the results of Ravichandran [10] stated above.

In the present paper, we investigate the differential operator

$$
a \frac{z f^{\prime}(z)}{\phi(g(z))}+b\left(1+\frac{z f^{\prime \prime}(z)}{f^{\prime}(z)}-\frac{z\left(\phi(g(z))^{\prime}\right.}{\phi(g(z))}\right)
$$

where $f, g \in \mathcal{A}$ and $\phi$ is an analytic function in a domain containing $g(\mathbb{E})$ such that $\phi(0)=0=\phi^{\prime}(0)-1$ and $\phi(w) \neq 0$ for $w \in g(\mathbb{E}) \backslash\{0\}$, for real numbers $a$ and $b(\neq 0)$. We, here, obtain some sufficient conditions for $\phi$-like, starlike and close-to-convex functions.

\section{Preliminaries}

We shall need the following definition and Lemmas to prove our main results.

Definition 1. [7, Def. 2.2b, p.21]. We denote by $Q$ the set of functions $p$ that are analytic and injective in $\overline{\mathbb{E}} \backslash \mathbb{B}(p)$, where

$$
\mathbb{B}(p)=\left\{\zeta \in \partial \mathbb{E}: \lim _{z \rightarrow \zeta} p(z)=\infty\right\},
$$

are such that $p^{\prime}(\zeta) \neq 0$ for $\zeta \in \partial \mathbb{E} \backslash \mathbb{B}(p)$.

Lemma 1. [7, Theorem 3.4h, p.132]. Let $q$ be univalent in $\mathbb{E}$ and let $\theta$ and $\varphi$ be analytic in a domain $\mathbb{D}$ containing $q(\mathbb{E})$, with $\varphi(w) \neq 0$, when $w \in q(\mathbb{E})$. Set $Q_{1}(z)=z q^{\prime}(z) \varphi[q(z)], h(z)=\theta[q(z)]+Q_{1}(z)$ and suppose that either

(i) $h$ is convex, or

(ii) $Q_{1}$ is starlike.

In addition, assume that

(iii) $\Re\left(\frac{z h^{\prime}(z)}{Q_{1}(z)}\right)>0$.

If $p$ is analytic in $\mathbb{E}$, with $p(0)=q(0), p(\mathbb{E}) \subset \mathbb{D}$ and

$$
\theta[p(z)]+z p^{\prime}(z) \varphi[p(z)] \prec \theta[q(z)]+z q^{\prime}(z) \varphi[q(z)],
$$

then $p(z) \prec q(z)$ and $q(z)$ is the best dominant. 
Lemma 2. [4]. Let $q$ be univalent in $\mathbb{E}$ and let $\theta$ and $\varphi$ be analytic in a domain $\mathbb{D}$ containing $q(\mathbb{E})$. Set $Q_{1}(z)=z q^{\prime}(z) \varphi[q(z)], h(z)=\theta[q(z)]+Q_{1}(z)$ and suppose that (i) $Q_{1}$ is starlike in $\mathbb{E}$ and

(ii) $\Re\left[\frac{\theta^{\prime}(q(z))}{\varphi(q(z))}\right]>0, z \in \mathbb{E}$.

If $p \in \mathcal{H}[q(0), 1] \cap Q$, with $p(\mathbb{E}) \subset \mathbb{D}$ and $\theta[p(z)]+z p^{\prime}(z) \varphi[p(z)]$ is univalent in $\mathbb{E}$ and

$$
\theta[q(z)]+z q^{\prime}(z) \varphi[q(z)] \prec \theta[p(z)]+z p^{\prime}(z) \varphi[p(z)], z \in \mathbb{E},
$$

then $q(z) \prec p(z)$ and $q(z)$ is the best subordinant.

\section{MAin RESUlts}

Theorem 3. Let $q, q(z) \neq 0$ be a univalent function in $\mathbb{E}$ and satisfies the condition

$$
\Re\left(1+\frac{z q^{\prime \prime}(z)}{q^{\prime}(z)}-\frac{z q^{\prime}(z)}{q(z)}\right)>\max \left\{0,-\frac{a}{b} \Re(q(z))\right\},
$$

where $a$ and $b(\neq 0)$ are real numbers. Let $\phi$ be analytic function in a domain containing $g(\mathbb{E}), \phi(0)=0=\phi^{\prime}(0)-1$ and $\phi(w) \neq 0$ for $w \in g(\mathbb{E}) \backslash\{0\}$. If $f, g \in$ $\mathcal{A}, \frac{z f^{\prime}(z)}{\phi(g(z))} \neq 0, z \in \mathbb{E}$, satisfy the differential subordination

$$
a \frac{z f^{\prime}(z)}{\phi(g(z))}+b\left(1+\frac{z f^{\prime \prime}(z)}{f^{\prime}(z)}-\frac{z\left(\phi(g(z))^{\prime}\right.}{\phi(g(z))}\right) \prec a q(z)+b \frac{z q^{\prime}(z)}{q(z)},
$$

then

$$
\frac{z f^{\prime}(z)}{\phi(g(z))} \prec q(z), \quad z \in \mathbb{E},
$$

and $q(z)$ is the best dominant.

Proof. Define the function $p(z)$ by

$$
p(z)=\frac{z f^{\prime}(z)}{\phi(g(z))} .
$$

Therefore

$$
\frac{z p^{\prime}(z)}{p(z)}=1+\frac{z f^{\prime \prime}(z)}{f^{\prime}(z)}-\frac{z\left(\phi(g(z))^{\prime}\right.}{\phi(g(z))}
$$

and (4) reduces to

$$
a p(z)+b \frac{z p^{\prime}(z)}{p(z)} \prec a q(z)+b \frac{z q^{\prime}(z)}{q(z)}
$$


Define $\theta$ and $\varphi$ as $\theta(w)=a w \& \varphi(w)=\frac{b}{w}$. Both $\theta$ and $\varphi$ are analytic in $\mathbb{C} \backslash\{0\}$ and $\varphi(w) \neq 0, w \in \mathbb{C} \backslash\{0\}$. Therefore $Q_{1}(z)=z q^{\prime}(z) \varphi(q(z))=b \frac{z q^{\prime}(z)}{q(z)}$ and

$$
h(z)=\theta(q(z))+Q_{1}(z)=a q(z)+b \frac{z q^{\prime}(z)}{q(z)} .
$$

A little calculation yields

$$
\frac{z Q_{1}(z)}{Q_{1}(z)}=1+\frac{z q^{\prime \prime}(z)}{q^{\prime}(z)}-\frac{z q^{\prime}(z)}{q(z)}
$$

and

$$
\frac{z h^{\prime}(z)}{Q_{1}(z)}=\frac{a}{b} q(z)+1+\frac{z q^{\prime \prime}(z)}{q^{\prime}(z)}-\frac{z q^{\prime}(z)}{q(z)}
$$

In view of Condition 3 , we have $Q_{1}(z)$ is starlike in $\mathbb{E}$ and $\Re\left(\frac{z h^{\prime}(z)}{Q_{1}(z)}\right)>0$. The proof, now, follows from the Lemma 1.

On taking $\phi(z)=z$ in Theorem 3, we have the following result:

Theorem 4. Let $q, q(z) \neq 0$, be a univalent function in $\mathbb{E}$, satisfying the Condition 3 of Theorem 3 for real numbers $a, b(\neq 0)$. If $f, g \in \mathcal{A}, \frac{z f^{\prime}(z)}{g(z)} \neq 0, z \in \mathbb{E}$, satisfy the differential subordination

$$
a \frac{z f^{\prime}(z)}{g(z)}+b\left(1+\frac{z f^{\prime \prime}(z)}{f^{\prime}(z)}-\frac{z g^{\prime}(z)}{g(z)}\right) \prec a q(z)+b \frac{z q^{\prime}(z)}{q(z)}
$$

then

$$
\frac{z f^{\prime}(z)}{g(z)} \prec q(z), z \in \mathbb{E},
$$

and $q(z)$ is the best dominant.

On taking $\phi(z)=z$ and $g(z)=f(z)$ in Theorem 3, we have the following result:

Theorem 5. Let $q, q(z) \neq 0$ be a univalent function in $\mathbb{E}$ and satisfies the Condition 3 of Theorem 3 for real numbers a and $b(\neq 0)$. If $f \in \mathcal{A}, \frac{z f^{\prime}(z)}{f(z)} \neq 0, z \in \mathbb{E}$, satisfies

$$
(a-b) \frac{z f^{\prime}(z)}{f(z)}+b\left(1+\frac{z f^{\prime \prime}(z)}{f^{\prime}(z)}\right) \prec a q(z)+b \frac{z q^{\prime}(z)}{q(z)}
$$

then

$$
\frac{z f^{\prime}(z)}{f(z)} \prec q(z), z \in \mathbb{E},
$$

and $q(z)$ is the best dominant. 
On selecting $a=1$ and $b=\alpha$ in the Theorem 5 , we get the following result for the class of $\alpha$-convex functions.

Theorem 6. Let $\alpha$ be a non zero real number and let $q, q(z) \neq 0$ be a univalent function in $\mathbb{E}$ satisfying the Condition 3 of Theorem 3. If $f \in \mathcal{A}, z \in \mathbb{E}$, satisfies

$$
(1-\alpha) \frac{z f^{\prime}(z)}{f(z)}+\alpha\left(1+\frac{z f^{\prime \prime}(z)}{f^{\prime}(z)}\right) \prec q(z)+\alpha \frac{z q^{\prime}(z)}{q(z)},
$$

then

$$
\frac{z f^{\prime}(z)}{f(z)} \prec q(z), z \in \mathbb{E},
$$

and $q(z)$ is the best dominant.

By defining $\phi(z)=g(z)=z$ in Theorem 3, we obtain the following result:

Theorem 7. Let $q, q(z) \neq 0$ be a univalent function in $\mathbb{E}$ and satisfying the Condition 3 of Theorem 3 for real numbers $a, b(\neq 0)$. If $f \in \mathcal{A}, f^{\prime}(z) \neq 0, z \in \mathbb{E}$, satisfies the differential subordination

$$
a f^{\prime}(z)+b \frac{z f^{\prime \prime}(z)}{f^{\prime}(z)} \prec a q(z)+b \frac{z q^{\prime}(z)}{q(z)}
$$

then

$$
f^{\prime}(z) \prec q(z), z \in \mathbb{E},
$$

and $q(z)$ is the best dominant.

Remark 1. It is easy to verify that dominant $q(z)=\left(\frac{1+z}{1-z}\right)^{\delta}, 0<\delta \leq 1$, satisfies the Condition 3 of Theorem 3, for real numbers $a$ and $b(\neq 0)$. Consequently, we get:

Theorem 8. Let $\phi$ be analytic function in the domain containing $g(\mathbb{E})$ such that $\phi(0)=0=\phi^{\prime}(0)-1$ and $\phi(w) \neq 0$ for $w \in g(\mathbb{E}) \backslash\{0\}$. If $f, g \in \mathcal{A}, \frac{z f^{\prime}(z)}{\phi(g(z))} \neq$ $0, z \in \mathbb{E}$, and for real numbers $a$ and $b(\neq 0)$, satisfy

$$
a \frac{z f^{\prime}(z)}{\phi(g(z))}+b\left(1+\frac{z f^{\prime \prime}(z)}{f^{\prime}(z)}-\frac{z\left(\phi(g(z))^{\prime}\right.}{\phi(g(z))}\right) \prec a\left(\frac{1+z}{1-z}\right)^{\delta}+\frac{2 b \delta z}{1-z^{2}},
$$

then

$$
\frac{z f^{\prime}(z)}{\phi(g(z))} \prec\left(\frac{1+z}{1-z}\right)^{\delta}, z \in \mathbb{E}, 0<\delta \leq 1
$$

On taking $\phi(z)=z$ in above theorem, we obtain: 
Corollary 9. Let $a$ and $b(\neq 0)$ are real numbers and $0<\delta \leq 1$. If $f, g \in$ $\mathcal{A}, \frac{z f^{\prime}(z)}{g(z)} \neq 0, z \in \mathbb{E}$, satisfy

$$
a \frac{z f^{\prime}(z)}{g(z)}+b\left(1+\frac{z f^{\prime \prime}(z)}{f^{\prime}(z)}-\frac{z g^{\prime}(z)}{g(z)}\right) \prec a\left(\frac{1+z}{1-z}\right)^{\delta}+\frac{2 b \delta z}{1-z^{2}}
$$

then

$$
\frac{z f^{\prime}(z)}{g(z)} \prec\left(\frac{1+z}{1-z}\right)^{\delta}, z \in \mathbb{E} .
$$

For $\phi(z)=z$ and $g(z)=f(z)$ in Theorem 8 , we obtain the following result:

Corollary 10. Let a and $b(\neq 0)$ are real numbers and $0<\delta \leq 1$. If $f \in \mathcal{A}, \frac{z f^{\prime}(z)}{f(z)} \neq$ $0, z \in \mathbb{E}$, satisfies the differential subordination

$$
(a-b) \frac{z f^{\prime}(z)}{f(z)}+b\left(1+\frac{z f^{\prime \prime}(z)}{f^{\prime}(z)}\right) \prec a\left(\frac{1+z}{1-z}\right)^{\delta}+\frac{2 b \delta z}{1-z^{2}},
$$

then

$$
\frac{z f^{\prime}(z)}{f(z)} \prec\left(\frac{1+z}{1-z}\right)^{\delta}, z \in \mathbb{E},
$$

and hence $f(z)$ is starlike.

Selecting $a=1$ and $b=\alpha$ in above corollary, we get the following result for the class of $\alpha$-convex functions:

Corollary 11. Let $\alpha$ be a non-zero real number. If $f \in \mathcal{A}, \frac{z f^{\prime}(z)}{f(z)} \neq 0, z \in \mathbb{E}$, satisfies

$$
(1-\alpha) \frac{z f^{\prime}(z)}{f(z)}+\alpha\left(1+\frac{z f^{\prime \prime}(z)}{f^{\prime}(z)}\right) \prec\left(\frac{1+z}{1-z}\right)^{\delta}+\frac{2 b \delta z}{1-z^{2}}
$$

then

$$
\frac{z f^{\prime}(z)}{f(z)} \prec\left(\frac{1+z}{1-z}\right)^{\delta}, z \in \mathbb{E}, 0<\delta \leq 1 .
$$

Hence $f(z)$ is strongly starlike.

On taking $\phi(z)=g(z)=z$ in Theorem 8 , we have: 
Corollary 12. Let $a$ and $b(\neq 0)$ are real numbers. If $f \in \mathcal{A}, f^{\prime}(z) \neq 0, z \in \mathbb{E}$, satisfies

$$
a f^{\prime}(z)+b \frac{z f^{\prime \prime}(z)}{f^{\prime}(z)} \prec a\left(\frac{1+z}{1-z}\right)^{\delta}+\frac{2 b \delta z}{1-z^{2}}
$$

then

$$
f^{\prime}(z) \prec\left(\frac{1+z}{1-z}\right)^{\delta}, z \in \mathbb{E}, 0<\delta \leq 1,
$$

and hence $f(z)$ is close-to-convex.

Remark 2. When we select the dominant $q(z)=e^{z}$, then this dominant satisfies the Condition 3 of Theorem 3 for non-zero real numbers $a$ and $b$ such that $\Re\left(e^{z}\right)>-\frac{b}{a}$. Consequently, we obtain the following result:

Theorem 13. Let $a$ and $b$ be non-zero real numbers such that $\Re\left(e^{z}\right)>-\frac{b}{a}$ and let $\phi$ be analytic function in a domain containing $g(\mathbb{E}), \phi(0)=0=\phi^{\prime}(0)-1$ and $\phi(w) \neq 0$ for $w \in g(\mathbb{E}) \backslash\{0\}$. If $f, g \in \mathcal{A}, \frac{z f^{\prime}(z)}{\phi(g(z))} \neq 0, z \in \mathbb{E}$, satisfy

$$
a \frac{z f^{\prime}(z)}{\phi(g(z))}+b\left(1+\frac{z f^{\prime \prime}(z)}{f^{\prime}(z)}-\frac{z\left(\phi(g(z))^{\prime}\right.}{\phi(g(z)}\right) \prec a e^{z}+b z
$$

then

$$
\frac{z f^{\prime}(z)}{\phi(g(z))} \prec e^{z}, z \in \mathbb{E}
$$

On choosing $\phi(z)=z$ in above theorem, we obtain:

Corollary 14. Let $a$ and $b$ non-zero real numbers such that $\Re\left(e^{z}\right)>-\frac{b}{a}$. If $f, g \in$ $\mathcal{A}, \frac{z f^{\prime}(z)}{g(z)} \neq 0, z \in \mathbb{E}$, satisfy the differential subordination

$$
a \frac{z f^{\prime}(z)}{g(z)}+b\left(1+\frac{z f^{\prime \prime}(z)}{f^{\prime}(z)}-\frac{z g^{\prime}(z)}{g(z)}\right) \prec a e^{z}+b z
$$

then

$$
\frac{z f^{\prime}(z)}{g(z)} \prec e^{z}, z \in \mathbb{E} .
$$

On selecting $\phi(z)=z$ and $g(z)=f(z)$ in Theorem 13, we get: 
Corollary 15. Let $a$ and $b$ are non-zero real numbers such that $\Re\left(e^{z}\right)>-\frac{b}{a}$. If $f \in \mathcal{A}, \frac{z f^{\prime}(z)}{f(z)} \neq 0, z \in \mathbb{E}$, satisfies the differential subordination

$$
(a-b) \frac{z f^{\prime}(z)}{f(z)}+b\left(1+\frac{z f^{\prime \prime}(z)}{f^{\prime}(z)}\right) \prec a e^{z}+b z
$$

then

$$
\frac{z f^{\prime}(z)}{f(z)} \prec e^{z}, z \in \mathbb{E},
$$

and hence $f(z)$ is starlike.

on choosing $a=1$ and $b=\alpha$ in above corollary, we obtain:

Corollary 16. Let $\alpha$ be a non-zero real number such that $\Re\left(e^{z}\right)>-\alpha$. If $f \in$ $\mathcal{A}, \frac{z f^{\prime}(z)}{f(z)} \neq 0, z \in \mathbb{E}$, satisfies

$$
\begin{gathered}
(1-\alpha) \frac{z f^{\prime}(z)}{f(z)}+\alpha\left(1+\frac{z f^{\prime \prime}(z)}{f^{\prime}(z)}\right) \prec e^{z}+\alpha z, \\
\frac{z f^{\prime}(z)}{f(z)} \prec e^{z}, \quad z \in \mathbb{E} .
\end{gathered}
$$

Therefore, $f \in \mathcal{S}^{*}$.

For $\phi(z)=g(z)=z$ in Theorem 13, we obtain the following result:

Corollary 17. Let $a$ and $b$ are non-zero real numbers such that $\Re\left(e^{z}\right)>-\frac{b}{a}$. If $f \in \mathcal{A}, f^{\prime}(z) \neq 0, z \in \mathbb{E}$, satisfies

$$
a f^{\prime}(z)+b \frac{z f^{\prime \prime}(z)}{f^{\prime}(z)} \prec a e^{z}+b z
$$

then

$$
f^{\prime}(z) \prec e^{z}, z \in \mathbb{E},
$$

and hence $f(z)$ is close-to-convex.

Remark 3. By selecting the dominant $q(z)=1+m z, 0<m \leq 1$, we observed that the Condition 3 of Theorem 3 holds for all real numbers a and $b(\neq 0)$ having same sign. Thus from Theorem 3, we have the following result: 
Theorem 18. Let $\phi$ be analytic function in the domain containing $g(\mathbb{E})$, where $\phi(0)=0=\phi^{\prime}(z)-1$ and $\phi(w) \neq 0$ for $w \in g(\mathbb{E}) \backslash\{0\}$. Let real numbers $a$ and $b(\neq 0)$ be such that $\frac{a}{b}>0$. If $f, g \in \mathcal{A}, \frac{z f^{\prime}(z)}{\phi(g(z))} \neq 0, z \in \mathbb{E}$, satisfy

$$
a \frac{z f^{\prime}(z)}{\phi(g(z))}+b\left(1+\frac{z f^{\prime \prime}(z)}{f^{\prime}(z)}-\frac{z\left(\phi(g(z))^{\prime}\right.}{\phi(g(z)}\right) \prec a(1+m z)+\frac{b m z}{1+m z}
$$

then

$$
\frac{z f^{\prime}(z)}{\phi(g(z))} \prec 1+m z, \text { where } 0<m \leq 1, z \in \mathbb{E} \text {. }
$$

Taking $\phi(z)=z$ in above theorem, we get the following result:

Corollary 19. Let $a$ and $b$ are non-zero real numbers having same sign and $0<$ $m \leq 1$. If $f, g \in \mathcal{A}, \frac{z f^{\prime}(z)}{g(z)} \neq 0, z \in \mathbb{E}$, satisfy

$$
a \frac{z f^{\prime}(z)}{g(z)}+b\left(1+\frac{z f^{\prime \prime}(z)}{f^{\prime}(z)}-\frac{z g^{\prime}(z)}{g(z)}\right) \prec a(1+m z)+\frac{b m z}{1+m z}
$$

then

$$
\frac{z f^{\prime}(z)}{g(z)} \prec 1+m z, z \in \mathbb{E} .
$$

From Theorem 18, for $\phi(z)=z$ and $g(z)=f(z)$, we obtain:

Corollary 20. Let $a$ and $b$ be non-zero real numbers having same sign and $0<$ $m \leq 1$. If $f \in \mathcal{A}, \frac{z f^{\prime}(z)}{f(z)} \neq 0, z \in \mathbb{E}$, satisfies

$$
(a-b) \frac{z f^{\prime}(z)}{f(z)}+b\left(1+\frac{z f^{\prime \prime}(z)}{f^{\prime}(z)}\right) \prec a(1+m z)+\frac{b m z}{1+m z},
$$

then

$$
\frac{z f^{\prime}(z)}{f(z)} \prec 1+m z, z \in \mathbb{E},
$$

and hence $f(z)$ is starlike.

On selecting $a=1$ and $b=\alpha$ in above corollary, we get the following result:

Corollary 21. For $\alpha>0$, if $f \in \mathcal{A}, \frac{z f^{\prime}(z)}{f(z)} \neq 0, z \in \mathbb{E}$, satisfies the differential subordination

$$
(1-\alpha) \frac{z f^{\prime}(z)}{f(z)}+\alpha\left(1+\frac{z f^{\prime \prime}(z)}{f^{\prime}(z)}\right) \prec(1+m z)+\frac{\alpha m z}{1+m z}
$$


then

$$
\frac{z f^{\prime}(z)}{f(z)} \prec 1+m z, \quad 0<m \leq 1,
$$

and hence $f(z)$ is starlike.

Selecting $\phi(z)=g(z)=z$, in Theorem 18, we have:

Corollary 22. Let a and $b(\neq 0)$ be real numbers having same sign. If $f \in \mathcal{A}, f^{\prime}(z) \neq$ $0, z \in \mathbb{E}$, satisfies

$$
a f^{\prime}(z)+b \frac{z f^{\prime \prime}(z)}{f^{\prime}(z)} \prec a(1+m z)+\frac{b m z}{1+m z}
$$

then

$$
f^{\prime}(z) \prec 1+m z, \quad 0<m \leq 1, \quad z \in \mathbb{E},
$$

and hence $f(z)$ is close-to-convex.

Remark 4. Let $q(z)=\frac{\beta(1-z)}{\beta-z}$, then

$$
\Re\left(1+\frac{z f^{\prime \prime}(z)}{f^{\prime}(z)}-\frac{z f^{\prime}(z)}{f(z)}\right)=\Re\left(\frac{\beta-z^{2}}{(\beta-z)(1-z)}\right)>0, \text { for } \beta>1
$$

and

$$
\Re q(z)=\Re\left(\frac{\beta(1-z)}{\beta-z}\right)>0 .
$$

In view of the above calculations, the Conditon 3 of Theorem 3 is satisfied for real numbers $a$ and $b(\neq 0)$ such that $\frac{a}{b}>0$. Consequently, we obtain the following result:

Theorem 23. Let $\phi$ be analytic function in the domain containing $g(\mathbb{E})$, where $\phi(0)=0=\phi^{\prime}(z)-1$ and $\phi(w) \neq 0$ for $w \in g(\mathbb{E}) \backslash\{0\}$. If $f \in \mathcal{A}, \frac{z f^{\prime}(z)}{\phi(g(z))} \neq 0, z \in \mathbb{E}$, for real numbers $a$, and $b(\neq 0)$ such that $\frac{a}{b}>0$, satisfies

$$
a \frac{z f^{\prime}(z)}{\phi(g(z))}+b\left(1+\frac{z f^{\prime \prime}(z)}{f^{\prime}(z)}-\frac{z\left(\phi(g(z))^{\prime}\right.}{\phi(g(z)}\right) \prec a \frac{\beta(1-z)}{\beta-z}+b \frac{(1-\beta) z}{(\beta-z)(1-z)}
$$

then

$$
\frac{z f^{\prime}(z)}{\phi(g(z))} \prec \frac{\beta(1-z)}{\beta-z}, z \in \mathbb{E}, \text { where } \beta>1 \text {. }
$$

Taking $\phi(z)=z$, we get the following result from above theorem: 
Corollary 24. If $f, g \in \mathcal{A}, \frac{z f^{\prime}(z)}{g(z)} \neq 0, z \in \mathbb{E}$, satisfy the differential subordination

$$
a \frac{z f^{\prime}(z)}{g(z)}+b\left(1+\frac{z f^{\prime \prime}(z)}{f^{\prime}(z)}-\frac{z g^{\prime}(z)}{g(z)}\right) \prec \frac{a \beta(1-z)}{\beta-z}+\frac{b(1-\beta) z}{(\beta-z)(1-z)},
$$

then

$$
\frac{z f^{\prime}(z)}{g(z)} \prec \frac{\beta(1-z)}{\beta-z}, z \in \mathbb{E},
$$

where $\beta>1$ and $a, b(\neq 0)$ are real numbers having same sign.

On selecting $\phi(z)=z$ and $g(z)=f(z)$ in Theorem 23, we obtain:

Corollary 25. Let $a$ and $b(\neq 0)$ be real numbers having same sign and $\beta>1$. If $f \in \mathcal{A}, \frac{z f^{\prime}(z)}{f(z)} \neq 0, z \in \mathbb{E}$, satisfies

$$
(a-b) \frac{z f^{\prime}(z)}{f(z)}+b\left(1+\frac{z f^{\prime \prime}(z)}{f^{\prime}(z)}\right) \prec \frac{a \beta(1-z)}{\beta-z}+\frac{b(1-\beta) z}{(\beta-z)(1-z)},
$$

then

$$
\frac{z f^{\prime}(z)}{f(z)} \prec \frac{\beta(1-z)}{\beta-z}, z \in \mathbb{E},
$$

and hence $f(z)$ is starlike.

Choosing $a=1$ and $b=\alpha$ in above corollary, we get:

Corollary 26. For $\alpha>0$, if $f \in \mathcal{A}, \frac{z f^{\prime}(z)}{f(z)} \neq 0, z \in \mathbb{E}$, satisfies the differential subordination

$$
(1-\alpha) \frac{z f^{\prime}(z)}{f(z)}+\alpha\left(1+\frac{z f^{\prime \prime}(z)}{f^{\prime}(z)}\right) \prec \frac{\beta(1-z)}{\beta-z}+\frac{\alpha(1-\beta) z}{(\beta-z)(1-z)},
$$

then

$$
\frac{z f^{\prime}(z)}{f(z)} \prec \frac{\beta(1-z)}{\beta-z}, \quad \beta>1, z \in \mathbb{E}
$$

i.e. $f \in \mathcal{S}^{*}$.

Taking $\phi(z)=g(z)=z$ in Theorem 23, we have: 
Corollary 27. Let $a, b(\neq 0)$ be real numbers having same sign and $\beta>1$. If $f \in \mathcal{A}, f^{\prime}(z) \neq 0, z \in \mathbb{E}$, satisfies

$$
a f^{\prime}(z)+b \frac{z f^{\prime \prime}(z)}{f^{\prime}(z)} \prec \frac{a \beta(1-z)}{\beta-z}+\frac{b(1-\beta) z}{(\beta-z)(1-z)},
$$

then

$$
f^{\prime}(z) \prec \frac{\beta(1-z)}{\beta-z}, z \in \mathbb{E},
$$

and hence $f(z)$ is close-to-convex.

Remark 5. On selecting the dominant $q(z)=1+\frac{2}{3} z^{2}$ in Theorem 3, it is easy to check that this dominant satisfies the Condition 3 of Theorem 3 for real numbers a and $b$ of same sign, as

$$
\Re\left(1+\frac{z f^{\prime \prime}(z)}{f^{\prime}(z)}-\frac{z f^{\prime}(z)}{f(z)}\right)=2 \Re\left(1+\frac{2}{3} z^{2}\right)^{-1}>0
$$

and

$$
\Re q(z)=\Re\left(1+\frac{2}{3} z^{2}\right)>0 .
$$

Consequently, we obtain the following result:

Theorem 28. For real numbers $a$ and $b(\neq 0)$ of same sign, if $f, g \in \mathcal{A}, \frac{z f^{\prime}(z)}{\phi(g(z))} \neq$ $0, z \in \mathbb{E}$, satisfy

$$
a \frac{z f^{\prime}(z)}{\phi(g(z))}+b\left(1+\frac{z f^{\prime \prime}(z)}{f^{\prime}(z)}-\frac{z\left(\phi(g(z))^{\prime}\right.}{\phi(g(z)}\right) \prec a\left(1+\frac{2}{3} z^{2}\right)+\frac{4 b z^{2}}{3+2 z^{2}},
$$

then

$$
\frac{z f^{\prime}(z)}{\phi(g(z))} \prec 1+\frac{2}{3} z^{2}, z \in \mathbb{E} .
$$

Here, $\phi$ is an analytic function in the domain containing $g(\mathbb{E})$, such that $\phi(0)=0=$ $\phi^{\prime}(z)-1$ and $\phi(w) \neq 0$ for $w \in g(\mathbb{E}) \backslash\{0\}$.

By selecting $\phi(z)=z$ in above theorem, we obtain:

Corollary 29. Let $a$ and $b(\neq 0)$ be real numbers such that $\frac{a}{b}>0$. If $f, g \in$ $\mathcal{A}, \frac{z f^{\prime}(z)}{g(z)} \neq 0, z \in \mathbb{E}$, satisfy

$$
a \frac{z f^{\prime}(z)}{g(z)}+b\left(1+\frac{z f^{\prime \prime}(z)}{f^{\prime}(z)}-\frac{z g^{\prime}(z)}{g(z)}\right) \prec a\left(1+\frac{2}{3} z^{2}\right)+\frac{4 b z^{2}}{3+2 z^{2}}
$$


then

$$
\frac{z f^{\prime}(z)}{g(z)} \prec 1+\frac{2}{3}, z^{2} z \in \mathbb{E} .
$$

On taking $\phi(z)=z$ and $g(z)=f(z)$ in Theorem 28, we have:

Corollary 30. Let a and $b(\neq 0)$ be real numbers such that $\frac{a}{b}>0$. If $f \in \mathcal{A}, \frac{z f^{\prime}(z)}{f(z)} \neq$ $0, z \in \mathbb{E}$, satisfies

$$
(a-b) \frac{z f^{\prime}(z)}{f(z)}+b\left(1+\frac{z f^{\prime \prime}(z)}{f^{\prime}(z)}\right) \prec a\left(1+\frac{2}{3} z^{2}\right)+\frac{4 b z^{2}}{3+2 z^{2}},
$$

then

$$
\frac{z f^{\prime}(z)}{f(z)} \prec 1+\frac{2}{3} z^{2}, z \in \mathbb{E}
$$

and hence $f(z)$ is starlike.

If we take $a=1$ and $b=\alpha$ in above corollary, we get:

Corollary 31. For $\alpha>0$, if $f \in \mathcal{A}, \frac{z f^{\prime}(z)}{f(z)} \neq 0, z \in \mathbb{E}$, satisfies the differential subordination

$$
(1-\alpha) \frac{z f^{\prime}(z)}{f(z)}+\alpha\left(1+\frac{z f^{\prime \prime}(z)}{f^{\prime}(z)}\right) \prec\left(1+\frac{2}{3} z^{2}\right)+\frac{4 \alpha z^{2}}{3+2 z^{2}},
$$

then

$$
\frac{z f^{\prime}(z)}{f(z)} \prec 1+\frac{2}{3} z^{2}, z \in \mathbb{E}
$$

and hence $f \in \mathcal{S}^{*}$.

In Theorem 28 , by selecting $\phi(z)=g(z)=z$, we obtain:

Corollary 32. Let real numbers $a$ and $b(\neq 0)$ be such that, $\frac{a}{b}>0$. If $f \in \mathcal{A}, f^{\prime}(z) \neq$ $0, z \in \mathbb{E}$, satisfies

$$
a f^{\prime}(z)+b \frac{z f^{\prime \prime}(z)}{f^{\prime}(z)} \prec a\left(1+\frac{2}{3} z^{2}\right)+\frac{4 b z^{2}}{3+2 z^{2}}
$$

then

$$
f^{\prime}(z) \prec 1+\frac{2}{3} z^{2}, z \in \mathbb{E},
$$

and hence $f(z)$ is close-to-convex. 


\section{SAndwich Type Results}

Theorem 33. Let $a$ and $b(\neq 0)$ be real numbers such that $\frac{a}{b}>0$. Let $q, q(z) \neq 0$ be univalent function in the unit disk $\mathbb{E}$, with $q(0)=1$ such that $\frac{z q^{\prime}(z)}{q(z)}$ is starlike univalent in $\mathbb{E}$ and $\Re q(z)>0$. Let $\phi$ be analytic function in the domain containing $g(\mathbb{E})$, where $\phi(0)=0=\phi^{\prime}(0)-1$ and $\phi(w) \neq 0$ for $w \in g(\mathbb{E}) \backslash\{0\}$. If $f, g \in \mathcal{A}$, $\frac{z f^{\prime}(z)}{\phi(g(z))} \in \mathcal{H}[q(0), 1] \cap Q$ with $\frac{z f^{\prime}(z)}{\phi(g(z))}\left(1+\frac{z f^{\prime \prime}(z)}{f^{\prime}(z)}-\frac{z\left(\phi(g(z))^{\prime}\right.}{\phi(g(z))}\right)$ is univalent in $\mathbb{E}$, satisfy

$$
a q(z)+b \frac{z q^{\prime}(z)}{q(z)} \prec a \frac{z f^{\prime}(z)}{\phi(g(z)}+b\left(1+\frac{z f^{\prime \prime}(z)}{f^{\prime}(z)}-\frac{z\left(\phi(g(z))^{\prime}\right.}{\phi(g(z)}\right),
$$

then

$$
q(z) \prec \frac{z f^{\prime}(z)}{\phi(g(z))}, z \in \mathbb{E},
$$

and $q(z)$ is the best subordinant.

Proof: Write $p(z)=\frac{z f^{\prime}(z)}{\phi(g(z))}$, then (5) becomes

$$
a q(z)+b \frac{z q^{\prime}(z)}{q(z)} \prec a p(z)+b \frac{z p^{\prime}(z)}{p(z)}
$$

By defining $\theta$ and $\varphi$ as $\theta(w)=$ aw and $\varphi(w)=\frac{b}{w}$, where $\theta$ and $\varphi$ are analytic in $\mathbb{C} \backslash\{0\}$ and $\varphi(w) \neq 0, w \in \mathbb{C} \backslash\{0\}$. Therefore,

$$
Q_{1}(z)=z q^{\prime}(z) \varphi(q(z))=b \frac{z q^{\prime}(z)}{q(z)} .
$$

A little calculation yields

$$
\frac{z Q_{1}(z)}{Q_{1}(z)}=1+\frac{z q^{\prime \prime}(z)}{q^{\prime}(z)}-\frac{z q^{\prime}(z)}{q(z)}
$$

and

$$
\frac{\theta^{\prime}(q(z))}{\varphi(q(z))}=\frac{a q(z)}{b}
$$

In view of the given conditions, $Q_{1}(z)$ is starlike and $\Re\left[\frac{\theta^{\prime}(q(z))}{\varphi(q(z))}\right]>0, z \in \mathbb{E}$. Hence the proof, now, follows from Lemma 2. 
Theorem 34. Let $q_{1}(z) \neq 0$ and $q_{2}(z) \neq 0$ be univalent in $\mathbb{E}$ such that $q_{1}(z)$ satisfies the condition of Theorem 33 whereas $q_{2}(z)$ satisfies the Condition 3 of Theorem 3. Let $\phi(z)$ be analytic function in the domain containing $g(\mathbb{E})$ such that $\phi(0)=0=$ $\phi^{\prime}(0)-1$ and $\phi(w) \neq 0$ for $w \in g(\mathbb{E}) \backslash\{0\}$. Let $f, g \in \mathcal{A}, \frac{z f^{\prime}(z)}{\phi(g(z))} \in \mathcal{H}[1,1] \cap Q$ and $a \frac{z f^{\prime}(z)}{\phi(g(z))}+b\left(1+\frac{z f^{\prime \prime}(z)}{f^{\prime}(z)}-\frac{z\left(\phi(g(z))^{\prime}\right.}{\phi(g(z))}\right)$ be univalent in $\mathbb{E}$, where a and $b(\neq 0)$ are real numbers. Further, if

$$
a q_{1}(z)+b \frac{z q_{1}^{\prime}(z)}{q_{1}(z)} \prec a \frac{z f^{\prime}(z)}{\phi(g(z))}+b\left(1+\frac{z f^{\prime \prime}(z)}{f^{\prime}(z)}-\frac{z\left(\phi(g(z))^{\prime}\right.}{\phi(g(z))}\right) \prec a q_{2}(z)+b \frac{z q_{2}^{\prime}(z)}{q_{2}(z)},
$$

then

$$
q_{1}(z) \prec \frac{z f^{\prime}(z)}{\phi(g(z))} \prec q_{2}(z), z \in \mathbb{E} .
$$

Moreover, $q_{1}(z)$ and $q_{2}(z)$ are the best subordinant and the best dominant respectively.

Taking $q_{1}(z)=1+m z$ and $q_{2}(z)=1+n z, 0<m<n \leq 1$, in Theorem 33, we have the following result:

Corollary 35. Let $\phi(z)$ be a analytic function in the domain containing $g \in \mathbb{E}$ such that $\phi(0)=0=\phi^{\prime}(0)-1$ and $\phi(w) \neq 0$ for $w \in g(\mathbb{E}) \backslash\{0\}$. Let $a, b(\neq 0)$ be real numbers such that $\frac{a}{b}>0$. If $f, g \in \mathcal{A}$ be such that $\frac{z f^{\prime}(z)}{\phi(g(z))} \in \mathcal{H}[1,1] \cap Q$ with $a \frac{z f^{\prime}(z)}{\phi(g(z))}+b\left(1+\frac{z f^{\prime \prime}(z)}{f^{\prime}(z)}-\frac{z\left(\phi(g(z))^{\prime}\right.}{\phi(g(z))}\right)$ is univalent in $\mathbb{E}$ and satisfy $a(1+m z)+\frac{b m z}{1+m z} \prec a \frac{z f^{\prime}(z)}{\phi(g(z))}+b\left(1+\frac{z f^{\prime \prime}(z)}{f^{\prime}(z)}-\frac{z\left(\phi(g(z))^{\prime}\right.}{\phi(g(z))}\right) \prec a(1+n z)+\frac{b n z}{1+n z}$ then

$$
1+m z \prec \frac{z f^{\prime}(z)}{\phi(g(z))} \prec 1+n z, \quad z \in \mathbb{E},
$$

where $m$ and $n$ are real numbers, such that $0<m<n \leq 1$.

On selecting $m=1 / 4, n=1 / 2$ and $a=1=b$ in above corollary, we obtain:

Example 1. Let $\phi(z)$ be a analytic function in the domain containing $g(\mathbb{E})$, where $\phi(0)=0=\phi^{\prime}(0)-1$ and $\phi(w) \neq 0$ for $w \in g(\mathbb{E}) \backslash\{0\}$. Let $f, g \in \mathcal{A}$ be such that $\frac{z f^{\prime}(z)}{\phi(g(z))} \in \mathcal{H}[1,1] \cap Q$ with $1+\frac{z f^{\prime}(z)}{\phi(g(z))}+\frac{z f^{\prime \prime}(z)}{f^{\prime}(z)}-\frac{z\left(\phi(g(z))^{\prime}\right.}{\phi(g(z))}$ is univalent in $\mathbb{E}$, and satisfy

$$
\frac{z}{4}+\frac{z}{4+z} \prec \frac{z f^{\prime}(z)}{\phi(g(z))}+\frac{z f^{\prime \prime}(z)}{f^{\prime}(z)}-\frac{z\left(\phi(g(z))^{\prime}\right.}{\phi(g(z))} \prec \frac{z}{2}+\frac{z}{2+z}
$$


then

$$
1+\frac{z}{4} \prec \frac{z f^{\prime}(z)}{\phi(g(z))} \prec 1+\frac{z}{2}, z \in \mathbb{E} .
$$

In Example 1, on taking $\phi(z)=z$, we get:

Example 2. Let $f, g \in \mathcal{A}$ be such that $\frac{z f^{\prime}(z)}{g(z)} \in \mathcal{H}[1,1] \cap Q$ with $1+\frac{z f^{\prime}(z)}{g(z)}+$ $\frac{z f^{\prime \prime}(z)}{f^{\prime}(z)}-\frac{z g^{\prime}(z)}{g(z)}$ is univalent in $\mathbb{E}$ and satisfy

$$
\frac{z}{4}+\frac{z}{4+z} \prec \frac{z f^{\prime}(z)}{g(z)}+\frac{z f^{\prime \prime}(z)}{f^{\prime}(z)}-\frac{z g^{\prime}(z)}{g(z)} \prec \frac{z}{2}+\frac{z}{2+z}
$$

then

$$
1+\frac{z}{4} \prec \frac{z f^{\prime}(z)}{g(z)} \prec 1+\frac{z}{2}, z \in \mathbb{E} .
$$

On selecting $\phi(z)=z$ and $g(z)=f(z)$ in Example 1, we get:

Example 3. Suppose $f \in \mathcal{A}$ is such that $\frac{z f^{\prime}(z)}{f(z)} \in \mathcal{H}[1,1] \cap Q$ with $1+\frac{z f^{\prime \prime}(z)}{f^{\prime}(z)}$ is univalent in $\mathbb{E}$ and satisfies

$$
\frac{z}{4}+\frac{z}{4+z} \prec \frac{z f^{\prime \prime}(z)}{f^{\prime}(z)} \prec \frac{z}{2}+\frac{z}{2+z}
$$

then

$$
1+\frac{z}{4} \prec \frac{z f^{\prime}(z)}{f(z)} \prec 1+\frac{z}{2}, z \in \mathbb{E}
$$

On taking $\phi(z)=g(z)=z$ in Example 1, we have:

Example 4. Suppose $f \in \mathcal{A}$ is such that $f^{\prime}(z) \in \mathcal{H}[1,1] \cap Q$ with $f^{\prime}(z)+\frac{z f^{\prime \prime}(z)}{f^{\prime}(z)}$ is univalent in $\mathbb{E}$ and satisfies

$$
1+\frac{z}{4}+\frac{z}{4+z} \prec f^{\prime}(z)+\frac{z f^{\prime \prime}(z)}{f^{\prime}(z)} \prec 1+\frac{z}{2}+\frac{z}{2+z}
$$

then

$$
1+\frac{z}{4} \prec f^{\prime}(z) \prec 1+\frac{z}{2}, z \in \mathbb{E}
$$




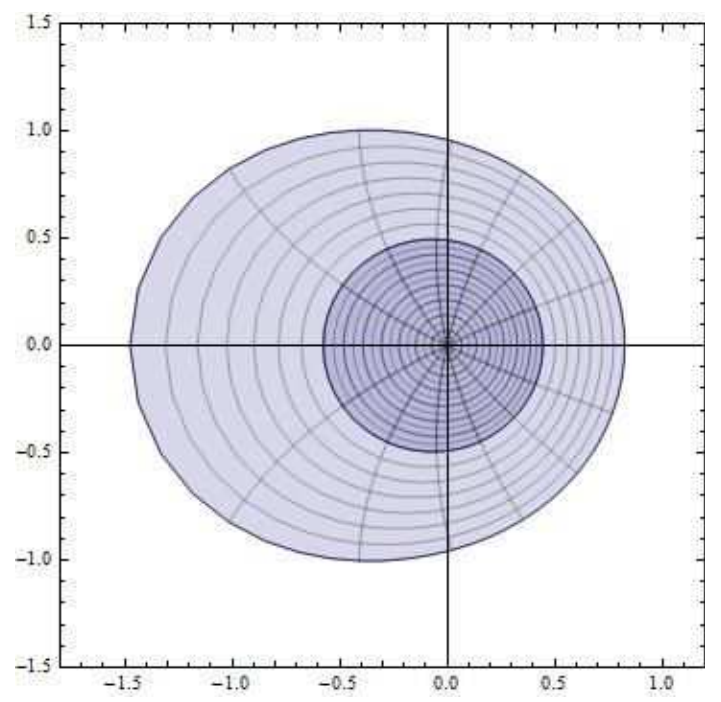

Figure 1

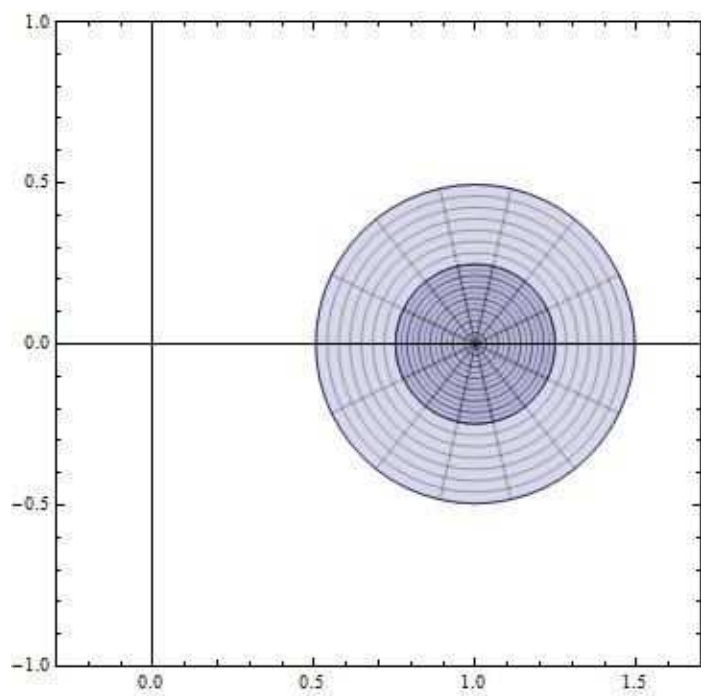

Figure 2

Using Mathematica 10.0, we plot the images of the unit disk under the functions $\frac{z}{4}+\frac{z}{4+z}$ and $\frac{z}{2}+\frac{z}{2+z}$ of (6) in Figure 1 and $1+\frac{z}{4}$ and $1+\frac{z}{2}$ of (7) in Figure 2. It follows that if $\frac{z f^{\prime}(z)}{\phi(g(z))}+\frac{z f^{\prime \prime}(z)}{f^{\prime}(z)}-\frac{z\left(\phi(g(z))^{\prime}\right.}{\phi(g(z))}$ takes values in the light shaded portion 
of Figure 1, then $\frac{z f^{\prime}(z)}{\phi(g(z))}$ will take values in the light shaded portion of Figure 2. Consequently, in view of Example 3 and Example $4, f$ is starlike and close to convex respectively.

\section{REFERENCES}

[1] L.Brickman, $\phi$-like functions I, Bull. Amer. Math. Soc. 79(1973), 555-558.

[2] K. Noshiro, On the theory of schlicht functions, J. Fac. Sci., Hokkaido Univ. 2(1934-35), 129-155.

[3] Rabha W. Ibrahim, On certain univalent class associated with first order differential subordinations, Tamkang Journal of Mathematics 42, 4(2011), 445-451.

[4] T. Bulboaca, Classes of first order Differential superordination-preserving integral operators, Demonstratio Mathematica 35, 2(2002), 287-292.

[5] T.N. Shanmugam, S. Sivassubramanian and Maslina Darus, Subordination and superordination results for $\phi$-like functions, Journal of ineq. in pure and applied mathematics 8, 1(2007), Art.20,6pp

[6] S. E. Warchawski, On the higher derivatives at the boundary in conformal mappings, Trans. Amer. Math. Soc. 38, 2 (1935), 310-340.

[7] S. S. Miller and P. T. Mocanu, Differential Subordinations: Theory and Applications, Marcel Dekker, New York and Basel, (2000).

[8] S. S. Miller, P. T. Mocanu and M. O. Reade, All $\alpha$ - convex functions are univalent and starlike, Proc. Amer. Math. Soc. 37, (1973), 553-554.

[9] St. Ruscheweyh, A subordination theorem for $\phi$-like functions, J. London Math. Soc. 2, 13 (1976), 275-280.

[10] V. Ravichandran, N. Mahesh and R. Rajalakshmi, On Certain Applications of Differential Subordinations for $\phi$-like Functions, Tamkang J. Math. 36, 2 (2005), 137-142.

Pardeep kaur

Department of Applied Sciences,

Baba Banda Singh Bahadur Engineering College, Fatehgarh Sahib-140407, Punjab, India.

e-mail: aradhitadhiman@gmail.com

Sukhwinder Singh Billing

Department of Mathematics, 
P. Kaur, S. Singh Billing - Some sandwich-type results ...

Sri Guru Granth Shaib World University, Fatehgarh Sahib-140407, Punjab, India. e-mail:ssbilling@gmail.com 